\title{
Case - Highly aggressive urothelial carcinoma of the bladder presenting with solitary metastasis to the phalanx
}

Hisham Assem, MD ${ }^{1}$; Nolan Broeke, $\mathrm{MSc}^{2}$; Lance Coleman, MPAS ${ }^{3}$; Geoffrey Gotto, $\mathrm{MD}^{2,3}$; Tarek A. Bismar, MD $^{1}$

${ }^{1}$ Department of Pathology and Laboratory Medicine, University of Calgary Cumming School of Medicine, Calgary, $\mathrm{AB}$, Canada; ${ }^{2}$ Prostate Cancer Centre, Calgary, AB; ${ }^{3}$ Department of Urology, University of Calgary, Cumming School of Medicine, Calgary, AB

Cite as: Can Urol Assoc J 2020 June 5; Epub ahead of print. http://dx.doi.org/10.5489/cuaj.6263

Published online June 5, 2020

$* * *$

\section{Introduction}

In the United States in 2019, it was projected that there would be 80,470 new cases of urinary bladder cancer diagnosed, with 17670 cancer-related deaths ${ }^{1}$. The ureters and other urinary organs accounted for approximately 4,000 cancer diagnoses in $2019^{1}$. Furthermore, these numbers do not include the relatively rare cancer diagnosis in the renal pelvis. Urothelial carcinomas include cancers of the bladder, ureters, and renal pelvis. When invasive, urothelial carcinomas typically extend locally with metastatic spread via lymphatics extending to the regional lymph nodes, liver, lungs, bones, and peritoneum. Muscle-invasive urothelial carcinomas are highly aggressive compared to cancers of the upper urinary tract, carrying a 5year disease specific survival rate of $<50 \%$ in pT2/pT3 disease and this survival rate drops below $10 \%$ in pT4 cancer ${ }^{2}$.

Metastatic carcinoma to the digits of the hands is exceedingly rare, although it has been reported. One such case reported metastasis of bronchogenic lung cancer to the fifth metacarpal ${ }^{3}$. We report a case of a 59-year-old male patient who presented with urothelial carcinoma of the bladder without evidence of metastatic disease on whole-body imaging. Pre-operative assessment noted redness and swelling of the right-fourth proximal phalange with involvement of the PIP joint, which was felt to be secondary to gout. He underwent right-laparoscopic nephrectomy, cystectomy and bilateral lymph node dissection. Post-operatively, swelling and pain of the finger worsened. X-rays demonstrated progression of erosive disease necessitating amputation of the digit. Histology of the amputated finger revealed metastatic urothelial carcinoma, consistent with a solitary metastasis at the time of presentation. Subsequent follow-up 
imaging, performed two-months after his initial imaging, confirmed widespread metastatic disease.

\section{Case presentation}

A 59-year-old male presented to a community hospital complaining of several weeks of gross hematuria. The patient smoked for 15-20 years, but never heavily and he presented with no cardiovascular or respiratory symptoms. Patient was anemic with a BMI of 26.8. Overall, the patient seemed to be in good general health when he presented to the hospital. Cystoscopy revealed significant tumor burden at the bladder-neck, -floor, -trigone, and right-lateral wall. Initial whole-body computed tomography (CT) imaging demonstrated a posterior bladder wall mass, with bilateral involvement of the uretrovesical junctions and bilateral hydroureteronephrosis with an atrophic right kidney. Extension of the tumor into the distal-right ureter was seen, in addition to suspected right seminal vesicle tumor involvement. Several mildly enlarged right internal and external iliac chain lymph nodes were noted, but otherwise no definite findings of metastatic disease were identified (Internal: $3.0 \times 2.2 \mathrm{~cm}$, External: $2.5 \times 2.5 \mathrm{~cm}$ ). His admission was complicated by acute renal failure secondary to obstruction, necessitating placement of bilateral percutaneous nephrostomies. Once stabilized, he underwent a diagnostic transurethral resection of bladder tumor (TURBT), with histology showing invasive high-grade papillary urothelial carcinoma with muscularis propria invasion and no lymphovascular invasion identified (pT2pN0, G2). He was subsequently transferred to a tertiary center for further management with, in chronological order: cystectomy, urinary diversion, TURBT, rightlaparoscopic nephroureterectomy, and bilateral lymph node dissection with ileal conduit.

During pre-operative assessment, it was noted that the patient's right-fourth proximal phalanx was red and swollen with involvement of the proximal interphalangeal (PIP) joint. His medical history was significant for osteoarthritis, primarily affecting the hand and feet, in addition to gout. He had experienced pain and swelling involving this digit over a period of 3-4 weeks, which did not resolve after a trial of antibiotics in the community. X-rays showed softtissue swelling, with interval development of erosive changes centered over the PIP joint on the right-fourth digit extending to the distal aspect of the proximal phalanx on a background of stable osteoarthritic changes involving the hands and feet. He was assessed by infectious disease, rheumatology and plastic surgery. Synovial fluid cultures were negative and a diagnosis of chronic gout/inflammatory arthropathy was rendered and he was treated with colchicine and allopurinol.

Due to his renal dysfunction, no pre-operative neoadjuvant chemotherapy was administered. A renal perfusion scan demonstrated significantly diminished function of the right kidney. Given the suspected involvement of the right-distal ureter he underwent a right laparoscopic nephroureterectomy in addition to radical cystoprostatectomy with ileal conduit formation. Pathology revealed high-grade invasive papillary urothelial carcinoma, with invasion into the prostate and a positive left pelvic lymph node (pT4aN1). 
Post-operatively, his right-forth digit was reassessed due to worsening pain and swelling. There was concern for osteomyelitis, and repeat X-rays demonstrated disease progression with complete obliteration of the PIP joint and extensive regional bone resorption. He was reassessed by plastic surgery and it was decided that amputation of the right-fourth finger was the best course of action. This was done without complication approximately two-weeks following his nephroureterectomy/radical cystoprostatectomy.

The resected digit consisted of the distal two-thirds of the right-fourth digit, measuring up to $7.0 \times 3.0 \times 1.0 \mathrm{~cm}$. Serial sectioning of the digit revealed a firm, well-circumscribed, white mass invading through bone and soft tissues measuring up to $4.0 \times 3.0 \mathrm{~cm}$ (Figs. 1A-B). Areas of calcification were also grossly identified. Portions of the mass were processed in alcohol to assess for gout as well as routine formalin fixation. Hematoxylin-Eosin stained slides revealed malignant nests and interconnected islands of high-grade squamoid-like cells extending through the dermis to the subcutaneous fat with focal involvement of the epidermis (Figs. 2A-D). Mitotic figures were readily identified. Immunohistochemistry (IHC), performed on formalin fixed paraffin embedded tissue, demonstrated tumor cells to be strongly and diffusely positive for pancytokeratin (AE1/AE3/CAM5.2), CK 5/6, 34ßE12, EMA, p63, Cyclin-D1, p53 and GATA 3. Ki67 demonstrated a high proliferative-index. Melan-A, S100, SOX 10 and NKX3.1 were all negative. Figs. 2E-H shows representative IHC images. Overall, the morphologic features and IHC results were consistent with a diagnosis of metastatic high-grade urothelial carcinoma. A portion of this tissue, processed through alcohol, showed needle-like crystals demonstrating negative birefringence consistent with gouty tophi.

A repeat whole-body CT-scan was subsequently preformed confirming extensive lung metastases, left adrenal metastasis, and right-axillary, pre-aortic, intra-abdominal, para-aortic and bilateral iliac metastatic lymphadenopathy. A suspected liver metastatic lesion in addition to innumerable lytic osseous metastasis was also present. Furthermore, imaging suggested inferior vena cava, right iliac and common femoral vein thrombosis. He experienced a rapid decline in his functional status and was seen by palliative medicine. The patient died approximately 3 months following his first presentation.

\section{Discussion}

In a series of 367 patients with muscle-invasive bladder cancer (pT2-4), 68\% were found to have metastases at autopsy ${ }^{4}$. Metastases are most commonly found in regional lymph nodes, followed by the liver, lung, bone, peritoneum, pleura, kidney, adrenal glands and intestine ${ }^{5}$. Outside of the pelvis, skeletal bones most frequently harbor urothelial carcinoma metastasis. Several case reports have described skeletal muscle metastasis, although this is considered rare ${ }^{6-8}$. As reported in this case, metastasis of urothelial carcinoma to the bones and soft tissues of the digits is exceedingly rare ${ }^{9}$. Subcutaneous tissue metastases of urothelial carcinoma have been previously described, although they typically involve the abdomen and perineum ${ }^{10}$. However, metastasis 
typically develops metachronous to the initial bladder tumor, often months-to-years later. This patient presented with a very aggressive form of urothelial carcinoma. His initial diagnostic cystoscope revealed a bladder-neck tumor and subsequent CT-scan didn't demonstrate evidence of metastatic disease. Thus, his swollen phalanx at the time of presentation did in-fact represent a solitary metastasis, which would be an unusual pattern for metastasis for urothelial carcinoma. He underwent a laparoscopic-right radical nephrectomy, radical cystectomy, and pelvic node dissection. Histopathology confirmed high-grade papillary urothelial carcinoma invading into the prostate with involvement of one pelvic lymph node (pT4aN1). Surprisingly, a repeat CT-scan two-months after his initial imaging documented diffuse metastatic disease including extensive lung parenchymal metastases, right-axillary metastatic lymphadenopathy, para-aortic lymphadenopathy, intra-abdominal para-aortic and bilateral iliac metastatic lymphadenopathy, large-left adrenal metastases, suspected liver metastases, and innumerable lytic metastases in the bony calvarium, in keeping with highly aggressive disease. Generally, patients with advanced metastatic disease (pT4) have a poor prognosis, with a 5-year survival of $18 \%{ }^{11}$. Unfortunately, this patient succumbed to his disease within three-months of his initial presentation. Recent analysis of gene expression and IHC from cases of muscle invasive bladder cancer has suggested five tumor-cell phenotypes of advanced urothelial carcinoma ${ }^{12}$. Of note, by IHC, our case demonstrated diffuse strong expression of cyclin D1 (CCND1) and absence of p16 expression, which is a pattern of IHC labelling identified in the 'urothelial-like' tumor cell phenotype group $^{13}$. These tumors frequently show $9 \mathrm{p} 21(\mathrm{CDKN} 2 \mathrm{~A})$ deletions/mutations, particularly in cases of advanced urothelial carcinoma ${ }^{14}$, which may explain the rapid progression and aggressive disease in this patient. Additionally, this tumor was positive for CK5/6 supporting basal phenotype, while at the same time staining positive for GATA3 and cyclin D1 with absence of synaptophysin, as seen in the "GU tumor phenotype". Thus, it seems this tumor shows a mixed molecular type which may explain its rapid clinical progression and lethal outcome. In the setting of metastatic carcinoma, there is no specific IHC marker or panel to confirm urothelial differentiation ${ }^{15}$. Positive IHC labelling for GATA3, CK20, p63 and high molecular-weight cytokeratin (HMWCK) or cytokeratin 5/6 can be of value when urothelial carcinoma is in the differential diagnosis ${ }^{16}$.

\section{Conclusions}

In summary, we present an exceedingly rare case of urothelial carcinoma presenting with solitary metastasis to a phalanx and rapid progression of disease suggesting a highly-aggressive form of urothelial cancer. Several IHC markers may predict the likelihood of an aggressive tumor phenotype, which could be confirmed with molecular subtyping. In addition, this case demonstrates the importance of correlating clinical history and imaging in the assessment of an unusual site of metastatic disease. 


\section{References}

1. Siegel RL, Miller KD, Jemal A: Cancer statistics, 2019. CA Cancer J Clin 2019, 69:7-34.

2. Liao RS, Gupta M, Schwen ZR, et al.: Comparison of Pathological Stage in Patients Treated with and without Neoadjuvant Chemotherapy for High Risk Upper Tract Urothelial Carcinoma. J Urology 2018, 200:68-73.

3. Tzaveas A, Paraskevas G, Pazis I, et al.: Metastasis of bronchogenic carcinoma to the 5th metacarpal bone: a case report. Cases J 2008, 1:284.

4. Wallmeroth A, Wagner U, Moch H, et al.: Patterns of metastasis in muscle-invasive bladder cancer (pT2-4): An autopsy study on 367 patients. Urol Int 1999, 62:69-75.

5. Shinagare AB, Ramaiya NH, Jagannathan JP, et al.: Metastatic pattern of bladder cancer: correlation with the characteristics of the primary tumor. AJR Am J Roentgenol 2011, 196:117-122.

6. Ekici S, Ozen H, Gedikoglu G, et al.: Skeletal muscle metastasis from carcinoma of the bladder. Scand J Urol Nephrol 1999, 33:336-337.

7. Nabi G, Gupta NP, Gandhi D: Skeletal muscle metastasis from transitional cell carcinoma of the urinary bladder: clinicoradiological features. Clin Radiol 2003, 58:883885.

8. Guidi M, Fusetti C, Lucchina S: Skeletal Muscle Metastases to the Flexor Digitorum Superficialis and Profundus from Urothelial Cell Carcinoma and Review of the Literature. Case Rep Urol 2016, 2016:2387501.

9. Taleb C, Pelissier P, Choughri H: [Bladder urothelial carcinoma with acrometastasis: a case report and review of the literature]. Chir Main 2011, 30:136-139.

10. Chaohui Lisa Zhao RCG, Anna Ellermeier, Kammi Henriksen, Ali Amin: Unusual manifestations of secondary urothelial carcinoma. 2016. pp. 1-5.

11. Fossa SD, Sternberg C, Scher HI, et al.: Survival of patients with advanced urothelial cancer treated with cisplatin-based chemotherapy. Br J Cancer 1996, 74:1655-1659.

12. Sjodahl G: Molecular Subtype Profiling of Urothelial Carcinoma Using a SubtypeSpecific Immunohistochemistry Panel. Methods Mol Biol 2018, 1655:53-64.

13. Sjodahl G, Eriksson P, Liedberg F, et al.: Molecular classification of urothelial carcinoma: global mRNA classification versus tumour-cell phenotype classification. J Pathol 2017, 242:113-125.

14. Aine M, Eriksson P, Liedberg F, et al.: Biological determinants of bladder cancer gene expression subtypes. Sci Rep 2015, 5:10957.

15. Amin MB, Trpkov K, Lopez-Beltran A, et al.: Best practices recommendations in the application of immunohistochemistry in the bladder lesions: report from the International Society of Urologic Pathology consensus conference. Am J Surg Pathol 2014, 38:e20-34. 
16. Paner GP, Annaiah C, Gulmann C, et al.: Immunohistochemical evaluation of novel and traditional markers associated with urothelial differentiation in a spectrum of variants of urothelial carcinoma of the urinary bladder. Hum Pathol 2014, 45:1473-1482.

\section{Figures and Tables}

Fig. 1. (A) Transverse and (B) longitudinal sections of resected phalanx demonstrating irregular white mass invading soft tissue and bone.

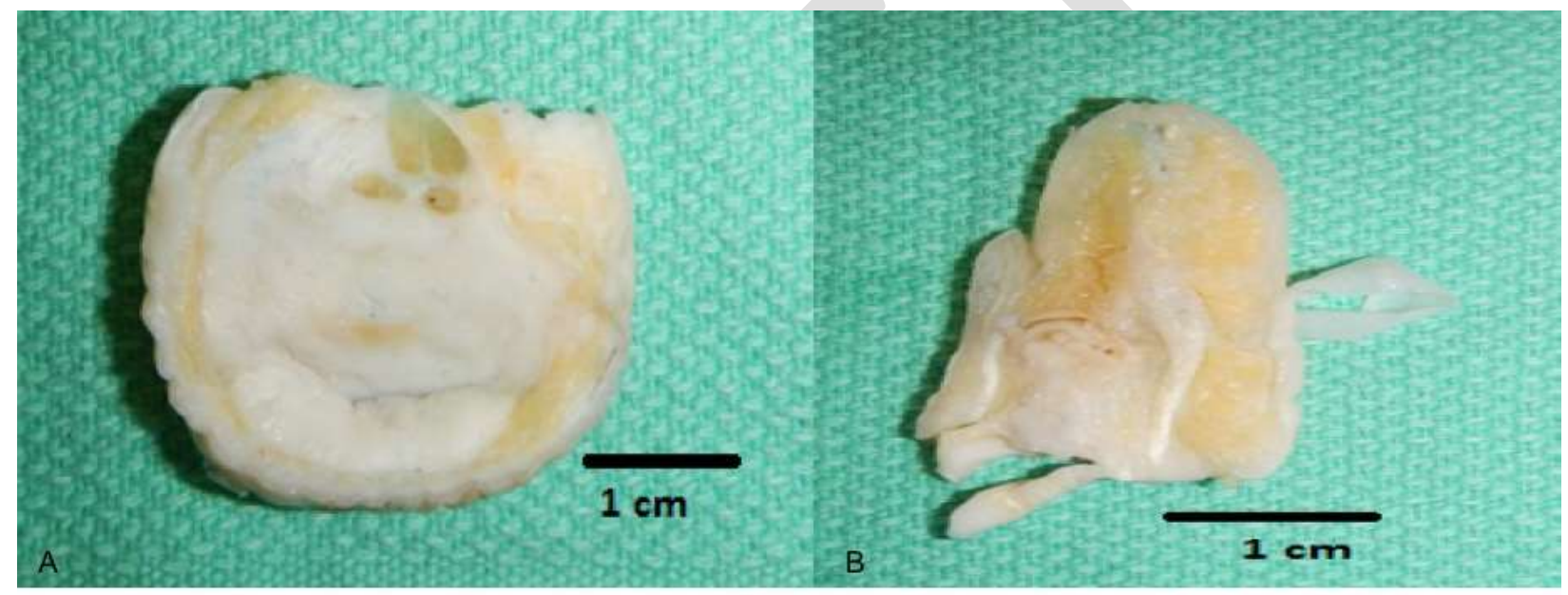

Fig. 2. (A) Histological examination of the resected phalanx revealed irregular tumor nests within the dermis (40x). (B) High power demonstrates the tumor composed of high-grade squamoid like cells (100x). (C) Urothelial carcinoma invading bone (x40). (D) Low-power view of high-grade squamoid urothelial carcinoma with associated comedo-like necrosis (x40). 


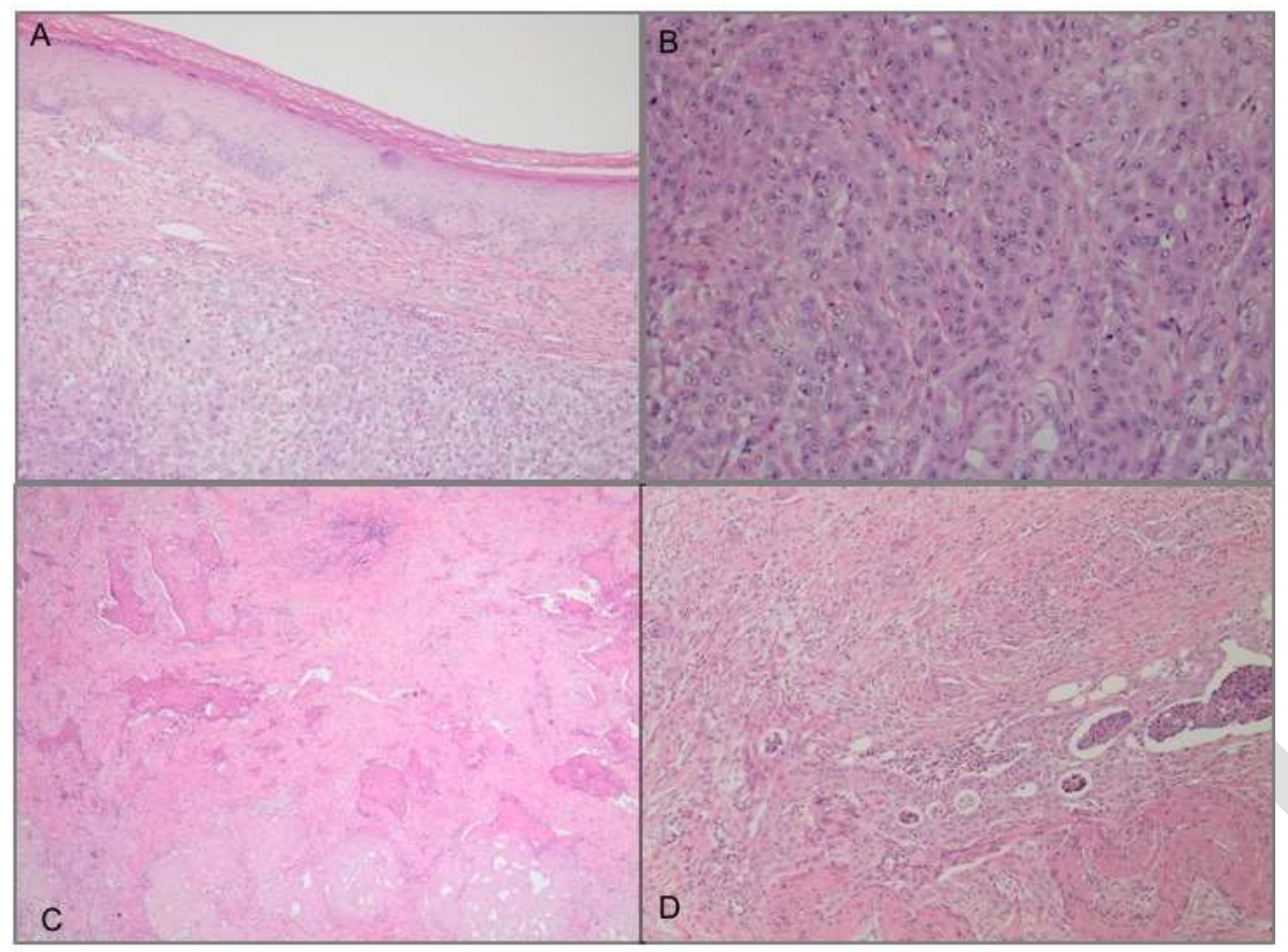

Fig. 2. (E) Immunohistochemistry demonstrated strong staining with high molecular weight cytokeratin (b34E12). (F) Expression of GATA3, consistent with metastatic urothelial carcinoma. (G) Cyclin-D1 expression. $(\boldsymbol{H})$ p53 wild-type expression 

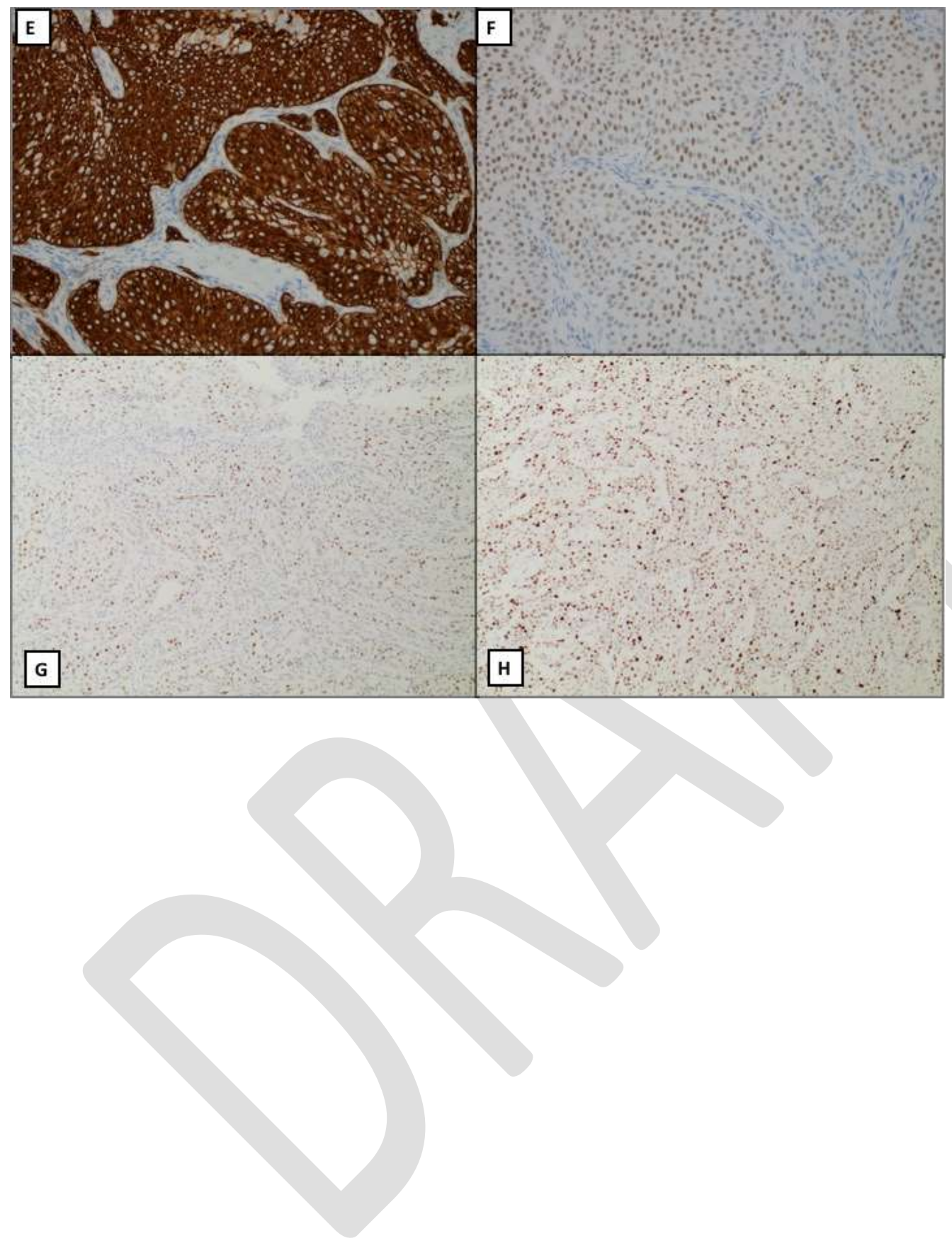